Sociologie et sociétés

\title{
Au-delà du choix rationnel : des sciences sociales plus politiques?
}

\section{Richard Balme}

Volume 34, numéro 1, printemps 2002

La théorie du choix rationnel contre les sciences sociales ? Bilan des débats contemporains

URI : https://id.erudit.org/iderudit/009749ar

DOI : https://doi.org/10.7202/009749ar

Aller au sommaire du numéro

\section{Éditeur(s)}

Les Presses de l'Université de Montréal

\section{ISSN}

0038-030X (imprimé)

1492-1375 (numérique)

Découvrir la revue

Citer ce document

Balme, R. (2002). Au-delà du choix rationnel : des sciences sociales plus politiques ? Sociologie et sociétés, 34(1), 101-112.

https://doi.org/10.7202/009749ar d'utilisation que vous pouvez consulter en ligne. 


\section{Au-delà du choix rationnel : des sciences sociales plus politiques?}

\section{RICHARD BALME}

Institut d'études politiques de Paris - CEVIPOF

27, rue Saint-Guillaume

75337 Paris Cédex 07, France

Courriel : richard.balme@sciences-po.fr

T A Discussion sur «le» choix rationnel proposée par J. B. Rule, J. L. Campell, D. D. Laitin et R. Boudon peut suggérer de nombreux commentaires sur des points précis de leurs argumentaires, au demeurant relativement différents les uns des autres. Ces contributions ont en commun leur position de critique constructive à l'égard du choix rationnel, avec laquelle je suis en sympathie, sans en partager nécessairement toutes les propositions. Au risque de négliger en partie l'intérêt intrinsèque de ces articles, je ne me livrerai pas à leur commentaire interne, peu approprié puisqu'il s'agit d'essais critiques adossés à des recherches originales exposées ailleurs. Il me semble plus pertinent de souligner un certain nombre de traits saillants pour en dégager les implications de mon point de vue de politiste européen. L'analyse des processus politiques d'une part, et les rapports entre les sciences sociales américaines et non américaines d'autre part, me paraissent en effet au cœur du débat suscité par le choix rationnel. Quelques observations générales sur ce point introduisent cette contribution. J'examine ensuite sommairement les apports du choix rationnel tels qu'ils se dégagent des quatre textes proposés, puis livre à la discussion deux questions critiques pour dépasser le choix rationnel : la reformulation éventuelle de l'axiomatique de rationalité, et la conciliation entre choix rationnel et néo-institutionnalisme. Enfin, la dernière section tente de préciser le statut du choix rationnel et ses différents usages dans les sciences sociales. 


\section{DE LA GUERRE CIVILE À LA PAX AMERICANA?}

Chacun de ces articles prend globalement la défense du choix rationnel. Défense contre quoi, et contre qui? D. Laitin parle de guerre de mouvement et de guerre de position pour définir la science politique américaine contemporaine, où l'affrontement entre «anciens» et «modernes» a laissé place à une pénétration générale des méthodes du choix rationnel (la modélisation formelle) dans les institutions académiques, au prix d'une reformulation substantielle de ses ambitions théoriques. Le mouvement par lequel les travaux fondateurs dans l'analyse économique des phénomènes politiques ont progressivement innervé la science politique, la sociologie et, dans une moindre mesure, la philosophie et la psychologie est assez connu. Les succès scientifiques et institutionnels de cette entreprise ont naturellement généré résistances et oppositions dans les secteurs des sciences sociales les plus directement menacés. C'est moins d'ailleurs les résultats substantiels de ces travaux que la validité de leurs méthodes — raisonnement axiomatique, modélisation déductive, et éventuellement modalités des tests empiriques - qui motive ce rejet. Celui-ci renvoie un écho lointain au refus de la «mathématisation » des sciences sociales qui accompagnait, dans les années 1960 et 1970 le développement des méthodes statistiques. Les approches les plus inductives et les plus relativistes, notamment ethnographiques, culturalistes ou historiques, ont été les plus rétives au développement du choix rationnel, en s'adossant généralement à une conception humaniste des sciences sociales, affirmant leur spécificité par rapport aux sciences de la nature, et notamment leur impossibilité de prédiction, et donc de modélisation. Souvent, l'argument se double d'un antiéconomisme, assimilant allègrement les emprunts de méthode à la microéconomie à l'hégémonie néolibérale. Les sociologues en général, les sociologues du politique en particulier, demeurent souvent arc-boutés sur la défense de leur territoire disciplinaire contre les économistes, les psychologues et les philosophes. Faut-il y voir la marque excessive d'un héritage durkheimien?

Si subsistent encore quelques combats isolés, dont certains sont fracassants ${ }^{1}$, cette guerre est néanmoins finie. Les sciences sociales les plus contemporaines font toutes une place importante aux apports des disciplines connexes. Mais les «succès» du choix rationnel ne signent pas non plus la domination de l'économie sur les autres sciences sociales. En termes d'objet d'abord, l'analyse économique fait l'aveu de son impuissance à rendre compte de l'ensemble du social en se cantonnant aux comportements de marché. Elle doit pour ce faire intégrer l'État, la démocratie électorale, les groupes d'intérêt, la loi, la démographie, les coutumes, et sortir ainsi d'un précarré devenu trop étroit. La théorie du choix rationnel est ici une nouvelle économie politique ${ }^{2}$, et on peut la comprendre comme une incursion de l'analyse politique, ou plus généralement institutionnelle, en économie autant que l'inverse. En termes théoriques ensuite, le programme initial formulé par les fondateurs les plus ambitieux du choix rationnel — tels

1. Voir, par exemple la critique au vitriol des Analytic Narratives de R. Bates, A. Greif, M. Levi, J. L. Rosenthal et B. Weingast par Jon Elster et la réplique des auteurs dans l'American Political Science Review, vol. $94, \mathrm{n}^{\circ} 3$, septembre 2000, p. 685-702.

2. Dont elle revendique d'ailleurs le titre dans le champ du public choice. 
Gordon Tullock, James Buchanan, William Riker ou Gary Becker — et correspondant aux conditions $\mathrm{P} 1$ à $\mathrm{P} 6$ spécifiées ici par $\mathrm{R}$. Boudon, se révèle faible si on le compare à ses propres attendus : les comportements empiriques de choix ne sont au mieux qu'approximativement rationnels; la modélisation ne permet pas toujours de définir un équilibre (paradoxe de l'électeur ou théorème du chaos); l'équilibre est éventuellement sous-optimal (dilemme du prisonnier); les situations sociales peuvent correspondre à des équilibres multiples non discernables par la théorie (dans le jeu Chicken par exemple). Le sociologue ne peut guère être perturbé par l'absence, la sous-optimalité ou la pluralité des équilibres générés par l'interaction sociale. Mais l'économiste en quête d'une théorie empirique universelle, précisément prédictive et prescriptive, doit ici en faire le deuil. Le bilan du choix rationnel dans le dernier demi-siècle (50 ans nous séparent de la première publication du Social Choice and Individual Values de K. Arrow) n'est donc pas, comme le prétendaient ses fondateurs, la dissolution des sciences sociales sous l'acide de l'économie néoclassique. C'est davantage celui de l'invention d'un paradigme commun à plusieurs d'entre elles, sans d'ailleurs qu'aucune ne puisse s'y réduire, ayant généré une méthodologie, des problématiques et des résultats suffisamment spécifiques et robustes pour provoquer le débat dont rend compte ce volume.

La première remarque que nous voudrions formuler ici concerne le caractère local de cette controverse. Le choix rationnel se range dans les «grandes théories» dont l'ambition est d'extraire la pensée sociale des contingences culturelles dans lesquelles elle se déploie. C'est même l'un des motifs principaux des résistances évoquées plus haut. Il est donc paradoxal de constater que ce débat se cantonne pour l'essentiel à l'espace scientifique et académique nord-américain. Des universitaires européens, sud-américains, indiens notamment apportent des contributions de valeur au courant du choix rationnel. Celui-ci est également utilisé pour traiter des questions sociales débordant largement le cadre américain contemporain. Mais c'est bien aux États-Unis qu'est produit l'essentiel des travaux de choix rationnel, que sont situés leurs vecteurs de diffusion et de légitimation, et que s'est déroulée la lutte académique évoquée précédemment, qui, sous cet angle, apparait comme une guerre civile américaine. En Europe, le choix rationnel n'est pas dans la même position d'hégémonie ou d'impérialisme. Il suffit de feuilleter une revue de sciences sociales pour constater que la modélisation formelle y est infiniment plus marginale. L'enjeu est plutôt ici pour le choix rationnel de sortir de son ghetto économiste et de s'imposer dans les départements et revues des autres sciences sociales, ce qu'il n'a pas vraiment réussi à faire jusqu'ici. L'Europe est aussi le théâtre de violentes guerres civiles, mais c'est plutôt autour des sociologies critiques et de leurs ambitions qu'a opéré la polarisation intellectuelle au cours des dernières années.

Cette situation est préoccupante pour la prétention des grandes théories et plus globalement pour les sciences sociales. Il n'existe aucune raison sérieuse pour que l'épistémologie des sciences sociales soit aujourd'hui différente des deux côtés de l'Atlantique, et pour qu'elle génère des débats incommensurables pendant des décennies entières. Puisque tel est néanmoins le cas, il faut en tirer les conséquences. Les sciences sociales sont-elles dépendantes de leur contexte d'énonciation au point d'être dans l'incapacité 
de formuler des énoncés extraits de celui-ci; et, du même coup, le projet théorique animant le choix rationnel est-il effectivement une chimère? C'est un argument souvent avancé par les opposants culturalistes ou relativistes au choix rationnel, que je ne partage pas. On peut imaginer que les conditions sociales de production des connaissances ont été suffisamment différentes entre l'Europe et la Chine prémodernes pour que la Renaissance inverse le leadership technologique entre les deux civilisations, et ouvre la voie au développement scientifique européen, comme le prétend Joseph Needham (1954). On peut également concevoir que l'État soviétique ait pesé sur l'agenda de recherche des mathématiciens et des physiciens russes pendant les années de communisme, ou que le système de financement de l'activité scientifique encourage la recherche appliquée aux États-Unis, peut-être au détriment de la recherche fondamentale. Mais si l'orientation des recherches est une chose, l'épistémologie en est une autre. Il n'y a pas de discontinuité dans les paradigmes des sciences naturelles entre les deux côtés de l'Atlantique, parce que les différences culturelles et institutionnelles sont trop insignifiantes pour donner lieu, au-delà des controverses «normales », à des conceptions scientifiques fondamentalement alternatives. Ce qui vaut entre L'Europe et les États-Unis vaut également pour le reste du monde, et ce qui vaut pour les sciences naturelles devrait valoir pour les sciences sociales. C'est par ailleurs tout le projet d'une compréhension réflexive du social qui est en jeu dans la capacité à formuler des propositions relativement indépendantes de la culture, et rejeter le choix rationnel à ce motif revient à renoncer à l'ambition scientifique de la pensée sociale.

L'autre raison pouvant expliquer cet écart transatlantique n'est plus le contexte social, mais au contraire la fermeture des milieux académiques fonctionnant en systèmes clos, nationaux et autoréférencés. Les Européens ne sont pas forcément les plus localistes dans cette perspective, puisque les sciences sociales américaines peuvent également rester très centrées sur la réalité nationale et cloisonnées sur elles-mêmes, peu ouvertes aux débats et aux enjeux sociaux étrangers. Quoi qu'il en soit, c'est un double déficit d'internationalisation et d'insertion sociale qui explique une telle anomalie. Cette interprétation est plus prosaïque que la précédente. Elle est plus sévère pour les institutions professionnelles qui portent la responsabilité de ce déficit scientifique. Mais elle a ma préférence, parce qu'elle ne scelle pas le destin des sciences sociales sous ce cloisonnement national, et laisse en vie leur vocation à élaborer des langages compréhensifs transculturels. Là encore cependant, le choix rationnel, si on le charge de cette pesante ambition d'universalisme, reste en-deçà de ses objectif, et moins performant que des théories plus limitées dans leurs objets mais plus globalement structurantes dans le débat scientifique international (la théorie psychosociale des attitudes, le néo-institutionnalisme, ou les théories de la globalisation par exemple).

\section{DE LA THÉORIE AU PARADIGME}

La Pax Americana n'a donc pas eu lieu. Pas plus que les grandes théories qui l'ont précédé, le choix rationnel n'a — heureusement!- pas réussi à unifier les sciences sociales dans la même approche de leur objet. Il est toutefois démesuré de l'évaluer à l'aune de 
cette ambition mégalomaniaque. On peut aussi observer qu'en dépit de ce tropisme nord-américain, le choix rationnel a généré des réflexions interdisciplinaires, des avancées sur les formes de l'explication dans les sciences sociales, des exigences accrues sur les modèles et leurs estimations empiriques, et au total a contribué à développer la qualité des recherches et la capacité critique à leur égard ${ }^{3}$. Quels sont donc ses apports essentiels, tels qu'ils ressortent des contributions rassemblées ici?

James Rule souligne d'abord que dans de très nombreux cas, le comportement social consiste effectivement en la poursuite de finalités relativement stabilisées par ajustement aux circonstances du choix et à l'information disponible. Ces situations dans lesquelles les gens font A plutôt que B, parce qu'ils préfèrent les conséquences estimées de $\mathrm{A}$ à celles de $\mathrm{B}$, semblent si fréquentes qu'elles retiennent peu l'attention des sociologues. Les comportements de consommation, de fréquentation ou d'activité sociale répondent très souvent à ce modèle simple. Celui-ci est abondamment mobilisé par la science politique, implicitement ou explicitement, lorsque le comportement d'un acteur - électeur, parti, État — est référé à son intérêt supposé. Ainsi, les commentaires électoraux les plus savants font-ils largement appel aux intentions et aux préférences des électeurs, telles que le résultat du scrutin est suppose les exprimer ${ }^{4}$. Il est de même difficile d'expliquer le comportement des partis politiques en campagne, ou celui des États en relations internationales, sans s'appuyer sur l'élucidation de leurs intérêts bien compris. C'est même l'un des fondements de «l'art» de la politique, depuis Machiavel et Hobbes. Sous cette forme, l'explication reste cependant à la fois triviale et irréfutable. Le choix rationnel permet donc de faire la part des choses, et de préciser ce qu'on explique, et ce qu'on n'explique pas, par l'intérêt. James Rule salue ainsi son rôle d'aiguillon sur les formulations théoriques alternatives ${ }^{5}$.

Un autre apport majeur du choix rationnel est sans doute d'avoir spécifié les modalités de l'explication en sciences sociales. Son fondement dans l'individualisme méthodologique a ainsi explicité les apories finalistes du fonctionnalisme, et plus généralement des raisonnements macrosociologiques ${ }^{6}$. Certains de ces principes méthodologiques sont évidemment énoncés par M. Weber ou E. Durkheim notamment, mais c'est pratiquement que les «boîtes noires» explicatives reliant, sans autre forme de procès, des variables à un niveau agrégé, ont subi une réfutation apparemment définitive sous les coups de boutoir de l'individualisme $e^{7}$ De même, le choix rationnel a convaincu (ou contraint?) nombre de recherches empiriques de recourir à des modèles explicatifs

3. Outre le débat récent sur Analytical Narratives, l'ouvrage de D. Green et I. Shapiro (1994) a également donné lieu à des controverses de grande tenue.

4. Alors même que les modèles de sociologie électorale dominants récusent l'idée d'un électeur rationnel...

5. On peut remarquer cependant que bien peu de ces alternatives empruntent la voie de la modélisation formelle. J'y reviendrai.

6. Plus qu'à l'économie, c'est à l'épistémologie de la sociologie qu'on doit cette contribution. On pense en particulier aux travaux de R. Boudon et de J. Elster.

7. Cette nécessaire introduction d'un niveau micro pour accéder à l'explication sociologique correspond à la mise en évidence de l'ecological fallacy dans l'analyse statistique, dont elle généralise le principe à l'ensemble des conceptualisations théoriques. 
intermédiaires, de portée plus générale que les seuls cas envisagés, en spécifiant les modes de relations entre les variables et les conditions de leur évaluation empirique.

Last but not least, et a contrario d'une idée assez répandue, le choix rationnel est une critique sévère de la pensée économique néoclassique. Cette critique est probablement la plus radicale qui soit, parce qu'elle est conçue dans les termes mêmes d'une discipline et d'une philosophie qui se révèle ainsi limitée pour la compréhension du social, et incapable de lui procurer ses fondements normatifs. Condorcet, Allais, Arrow, Olson, Downs, Riker, McKelvey ou Schofield ont tous attaché leur nom à la mise en évidence de paradoxes révélés par la modélisation d'interactions fondées sur les axiomes de liberté de préférences et de choix. Ces paradoxes peuvent être strictement théoriques (dans le social choice), ou empiriques (paradoxes de l'électeur ou de l'action collective). Leurs solutions résident toutes dans la restriction des préférences ou des procédures de choix par des facteurs sociaux (les valeurs, les normes, la structure des groupes), ou institutionnels (la réduction des alternatives soumises au choix, la restriction du nombre de leurs dimensions, l'organisation du programme ${ }^{8}$ ). L'intérêt majeur du choix rationnel est donc de spécifier, en prenant la mesure de l'écart avec un idéal de rationalité, quelles conditions sont nécessaires à des comportements coopératifs, à la participation à l'action collective, ou à une décision publique démocratique. Économie, science politique, philosophie, sociologie ont ainsi adopté un espace de réflexion commun, doté d'une même façon de poser les problèmes sociaux, sinon de les résoudre, structurant une large part de leurs débats. La constitution et la pérennité de ce paradigme est incontestablement un acquis important. Mais elle ne valide par pour autant la théorie du choix rationnel.

\section{FAUT-IL RENONCER A LA RATIONALITÉ ?}

L'une des principales critiques adressée à la théorie du choix rationnel concerne son axiomatique de rationalité. L'intérêt personnel se révélerait trop restrictif - et trop trivial - pour rendre compte de la complexité des comportements sociaux. Cette critique, adressée à une version restrictive du choix rationnel (thin rationality) porte à mon sens assez peu, parce qu'elle confond choix rationnel et utilitarisme. Le choix rationnel exige seulement que les individus aient des préférences. Si cette condition est contraignante, elle n'indique rien sur l'objet et la nature des préférences considérées. Celles-ci peuvent donc être morales ou altruistes plutôt que matérielles et égoïstes, sans affecter en aucune manière le raisonnement. On objecte souvent qu'une conception extensive de la rationalité (thick rationality), telle celle qui est proposée ici par $\mathrm{R}$. Boudon, donne un caractère tautologique à la théorie. Mais la diversité des motivations, c'est-à-dire des types de préférences, n'a aucune incidence sur la façon dont les individus opèrent effectivement leurs choix, en accord plus ou moins strict avec leurs préférences. Il est tout aussi spéculatif de penser que «chacun, agissant dans son intérêt propre, œuvre au bénéfice de tous» (proposition utilitariste) que «chacun, œuvrant

8. C’est l'objet de la littérature sur les «équilibres structurellement induits». 
dans l'intérêt de tous, agit rationnellement» (proposition idéaliste). Il est donc parfaitement légitime et heuristique d'explorer si, les préférences étant données, la rationalité permet de comprendre les formes agrégées de l'interaction. Ceci n'est pas acquis d'avance.

Considérons d'abord la trilogie avancée par R. Boudon entre rationalité instrumentale, rationalité cognitive et rationalité axiologique. Les premier et troisième types correspondent à la rationalité en finalité et à la rationalité en valeur chez Max Weber. La rationalité cognitive correspond aux motivations dictées non par ce qui est efficient (rationalité instrumentale) ou par ce qui est juste (rationalité axiologique), mais par ce qui est vrai, en tout cas considéré comme tel par l'acteur. Il s'agit donc de croyances pratiques, par opposition aux croyances normatives impliquées par le troisième type. L'intérêt de la typologie est d'élargir le chromatisme des intentions et des justifications de choix envisagées, en incorporant des dimensions plus sociologiques, notamment les notions de justice (fairness) et de justesse (appropriateness), qu'on retrouve dans le néo-institutionnalisme sociologique (March et Olsen, 1989). Cette conceptualisation est incontestablement plus satisfaisante que l'économisme de la thin rationality évoqué plus haut, comme le défend $\mathrm{R}$. Boudon. Mais sauve-t-elle l'axiome de rationalité pour autant?

Cela n'est pas certain. En effet, des croyances pratiques sont impliquées à la fois dans la rationalité instrumentale (l'acheteur rationnel se fonde par exemple sur une croyance pratique dans la valeur de la monnaie) et dans la rationalité axiologique (le choix de sauver l'honneur se fonde sur l'anticipation pratique du déshonneur). On peut donc rester réservé sur la proposition de constituer la cognition en une forme spécifique de rationalité. Il semble plus important de considérer les conditions sous lesquelles les croyances pratiques restent «rationnellement» indépendantes des préférences, ce qui renvoie à la rationalité limitée et à la dissonance cognitive. Dans un modèle de rationalité limitée, l'acteur optimalise la probabilité de réalisation de ses préférences sous contrainte d'information, c'est-à-dire à partir de ce qu'il sait ou ce qu'il croit vrai. En théorie des jeux, c'est-à-dire dans les contextes stratégiques, c'est un raisonnement bayesien qui est utilisé pour rendre compte de ce processus par lequel les stratégies et les croyances sont consistantes et se renforcent mutuellement. Le calcul de probabilités conditionnelles est certes éloigné de la psychologie empirique du choix. Mais la question est de savoir s'il fournit une métaphore du raisonnement acceptable pour en prédire les effets agrégés. Cela peut être relativement le cas dans les conditions de risque, mais plus douteux dans les conditions d'incertitude, où d'autres critères de choix peuvent plus probablement intervenir ${ }^{9}$. Il ne me semble pas illégitime de ramener dans certains cas, et peut-être dans les cas les plus stratégiques, les conditions d'incertitude à des conditions de risque. Mais aucune théorie ne spécifie précisément dans quelles circonstances cette réduction est acceptable.

9. Dans les conditions de risque, les décideurs ont la faculté d'attribuer des probabilités subjectives aux événements. Dans les conditions d'incertitude ce n'est plus le cas. 
Même dans les cas d'information parfaite, l'interaction entre préférences et croyances n'est pas nécessairement rationnelle. Élaborant la théorie de L. Festinger, Jon Elster a exploré certains mécanismes de réduction ou d'évitement de la dissonance cognitive, par exemple le wishful thinking (les préférences engendrent des croyances), ou les «raisins amers» (les préférences s'adaptent aux croyances) ${ }^{10}$. Enfin et peut-être encore plus au fond, les préférences elles-mêmes se révèlent irrationnelles, c'est-à-dire inconsistantes, notamment dans les évaluations asymétriques du risque (Tversky et Kahneman, 1974, p. 1124-1130) et dans l'incorporation hyperbolique plutôt qu'exponentielle du temps dans l'estimation des gains (Ainslie, 1992). Au total, la dimension cognitive invite moins à reconsidérer l'objet de la rationalité que son idée même, c'està-dire l'indépendance des préférences à l'égard des croyances pratiques. Cette relaxation de l'axiome de rationalité est peut-être moins un échec qu'un résultat de la théorie $\mathrm{du}$ choix rationnel, puisqu'elle en est en quelque sorte l'aboutissement. Mais il faut admettre qu'elle débouche sur un autre cadre interprétatif, où l'intentionnalité se révèle beaucoup plus complexe que la rationalité, et où la modélisation, quand elle reste praticable, exige d'autres postulats.

\section{CHOIX RATION NEL ET NÉO-INSTITUTION NALISME}

Sur un autre plan, la théorie du choix rationnel est mise en cause par le développement des approches néo-institutionnalistes dans les sciences sociales. On en trouve l'expression en économie, avec la nouvelle économie institutionnelle, en sociologie, avec des réflexions centrées sur la signification des institutions, et en science politique, avec les notions de trajectoires historiques et de path-dependency. Ces approches connaissent de nombreuses déclinaisons. Mais l'une de leurs plus communes et plus courantes assertions critique l'usage de la notion de préférence par le choix rationnel. Pour les néo-institutionnalistes, les individus ne sont pas libres de leurs préférences, et l'analyse sociologique ou politique ne saurait rester muette sur la genèse de celles-ci, comme l'économie prétend le faire. Les institutions créent les individus et leurs préférences, et non le contraire, comme dans l'approche du choix rationnel. John Campbell plaide ainsi pour une réintroduction des idées dans la réflexion, et distingue quatre types de représentations collectives susceptibles de générer des préférences : les paradigmes, les sentiments publics, les programmes politiques et les cadres interprétatifs. Sa typologie est habilement fondée sur la distinction entre les dimensions cognitives et normatives des idées d'une part, et entre leurs dimensions d'héritage (background) et d'anticipation (foreground) d'autre part. Cette approche est très stimulante pour l'analyse des processus politique et des politiques publiques en particulier. J. Campbell fait bien apparaître les difficultés méthodologiques considérables auxquelles se heurte le néo-institutionnalisme pour dépasser une pétition de principe aussi ancienne que la sociologie, mais dont le simple rappel ne fait guère progresser la réflexion. Si les institutions «font» les individus, ou si les idées (conçues à un niveau collectif) déterminent les préférences (au niveau individuel), il faut

10. Voir notamment Sour Grapes (1983). 
encore montrer par quels processus, comment différentes idées engendrent différents types de préférences, et comment les idées ou les institutions changent. Il n'y a effectivement aucun fondement théorique solide à l'opposition entre intérêts et idées, et la controverse est limitée par le fait qu'il n'est pas envisageable de réfuter le choix rationnel par le néo-institutionnalisme, puisque les préférences peuvent être axiologiques, et réciproquement, puisque la formation historique des préférences et des institutions n'empêche en rien leur usage stratégique. J. Campbell peut donc légitimement appeler à un dialogue plus constructif entre les deux courants, et en refusant de «choisir un camp», il offre une contribution stimulante à la réflexion sur l'articulation entre représentations collectives et processus politiques. Mais son approche reste à un niveau mésopolitique, sa typologie aidant à classifier des processus politiques globaux sans spécifier plus avant les formes d'interactions individuelles dont ils sont à la fois le site et le résultat. Cet effort me semble à la fois légitime et convaincant. Mais je crains cependant qu'il surestime les possibilités du choix rationnel, et qu'il en exige davantage qu'il ne peut produire. Le choix rationnel n'a pas de position ou d'instrument conceptuel pour aborder la formation des préférences. L'explication qu'il propose est toute entière fondée sur l'anticipation des interactions et de leurs conséquences. Si le choix rationnel a opéré une révolution dans les façons de penser la vie sociale et les formes de causalité qui lui sont associées, c'est bien celle qui consiste à expliquer le présent comme l'anticipation de l'avenir, et non comme l'incarnation du passé. Ce changement de perspective est considérable, dans la mesure ou la causalité suppose le plus souvent que l'explanans précède l'explanandum, et non l'inverse. Il exige aussi de considérer que les contraintes sociales actuelles résultent, pour une part qu'il se propose de révéler, des représentations que les acteurs se font de l'avenir, et ne sont pas le sédiment mécanique de l'histoire.

Cette conception sociologique est très weberienne, c'est-à-dire très centrée sur l'«activité sociale». Elle exige que les acteurs aient des préférences, mais elle est indifférente à leur orientation et à leur mode de constitution. C'est sans doute à ce prix que le choix rationnel est plus spécifique que la plupart des théories dans son domaine d'investigation qu'est l'interaction stratégique. Il est certes compatible avec n'importe quelle approche de la formation des préférences, puisqu'il les admet comme données. Mais il n'aide en rien à la compréhension de mécanismes qui ne sont pas son objet, et dont l'exploration se situe simplement sur une autre plan analytique. Ce faisant, c'est plutôt la limitation du domaine de validité du choix rationnel qu'on met en évidence ici que la définition de nouvelles perspectives de théorisation.

\section{THÉORIE, INDIVIDUALISME, MODÉLISATION}

Pour résumer les propos précédents, on peut dresser le constat suivant. Le développement du choix rationnel dans les dernières décennies représente la constitution d'un paradigme transdisciplinaire dont l'importance est considérable, sans doute le seul équivalent contemporain à ce que furent en leur temps le structuro-fonctionnalisme ou le behaviorisme. Ce paradigme a transformé les conceptualisations et les méthodes de très nombreuses disciplines. Et pourtant la théorie qu'il revendique, ou qui lui est imputée 
selon les cas, se révèle empiriquement fausse, ou au mieux limitée à un domaine de validité restreint. R. Boudon plaide ainsi pour l'individualisme méthodologique plutôt que pour la théorie du choix rationnel, et D. Laitin préfère, quant à lui, retenir le legs méthodologique de la modélisation formelle. Il est un peu hasardeux de spéculer sur l'avenir des sciences sociales, mais suffisamment d'éléments semblent rassemblés pour penser que le choix rationnel a vraisemblablement franchi son optimum heuristique, et produira désormais des résultats «à rendement décroissant». Ceci ne veut pas dire que ces apports puissent être ignorés ou globalement rejetés. C’est pourtant souvent le cas, essentiellement en raison de la diversité des usages scientifiques de ce paradigme, et de l'accumulation des malentendus qui en résulte. Essayons de distinguer quelques-uns de ces usages.

En premier lieu, l'ensemble du Social Choice, la théorie fondamentale des jeux et une partie du Public Choice forment le versant normatif de la théorie du choix rationnel, ici purement axiomatique et déductive, mais tout aussi légitime que les mathématiques ou la physique théorique par exemple, dont les canons de scientificité ne sont pas contestés au seul motif de leur caractère strictement analytique. Les sciences sociales sont aujourd'hui dominées par un empirisme qui confond souvent neutralité axiologique, objectivisme et "devoir de réserve», et les détourne — à tort — des dimensions normatives de la pensée sociale. Peu d'arguments strictement normatifs sont avancés pour critiquer le choix rationnel dans le domaine où il est peut être le moins exposé, et où il détermine analytiquement les propriétés formelles des interactions sociales et politiques. Ce qui lui vaut ici tant d'hostilité, c'est le principe même du raisonnement axiologique, qui fait d'un résultat déductif une quasi-prescription, selon les auteurs, implicite ou explicite. Les sciences sociales ont pourtant, avec le choix rationnel, le moyen d'explorer virtuellement les conséquences des arrangements institutionnels dans les contextes normativement fondés sur la rationalité, c'est-à-dire essentiellement le marché et la démocratie. Comment ne pas voir là un enjeu intellectuel majeur, à la fois sur les plans social et scientifique? Je ne vois aucune objection épistémologique valide au développement de ce type de raisonnement. Mais c'est surtout parce qu'il explore les implications normatives des institutions sociales contemporaines que l'incorporation de ses résultats semble résolument nécessaires à l'ensemble des disciplines.

L'autre versant du choix rationnel, l'essentiel de ses contributions en sociologie et science politique, est empirique. C'est généralement parce qu'il est peu prédictif empiriquement qu'on refuse au choix rationnel ses prétentions prescriptives dans le registre normatif. Mais prédictif de quoi? Le choix rationnel n'est assurément pas une psychologie du choix individuel. Il a été parfois revendiqué comme telle par ses promoteurs, mais cette position n'est plus tenable aujourd'hui, si tant est qu'elle l'ait jamais été. Cette psychologie est au mieux pauvre, et au pire fausse. C'est mal comprendre le raisonnement cependant que de focaliser l'estimation empirique sur les axiomes de la théorie. C'est bien le degré de validité du modèle, et non l'exactitude de l'axiomatique, qui doit guider l'évaluation de la théorie. Ces modèles sont par exemple la formalisation spatiale de la compétition électorale chez Anthony Downs, celle de la croissance bureaucratique chez W. Niskanen, ou l'ensemble des nombreux modèles d'interaction avancés par la théorie 
des jeux. Modéliser ainsi permet plusieurs types d'investigation empirique. Ce que connait en général le chercheur, c'est le résultat de l'interaction, telle qu'une situation durable de conflit, de coopération, ou d'asymétrie dans une relation. On peut donc inférer avec un postulat de rationalité la ou les structures de préférences, d'information et de décision qui donnent à une situation sociale son fondement comportemental. À charge de l'exploration empirique de vérifier la réalité ou la plausibilité de ces structures cognitives. (C’est la démarche revendiquée par les Analytical Narratives.) On peut aussi raisonner à l'inverse, en partant des préférences, ou en les supposant empiriquement connues, et par une projection rationnelle déduire les situations sociales qu'elles doivent «logiquement» produire. Empiriquement, ce sont alors ces situations qu'on estime plus précisément pour spécifier davantage les préférences et la structure du choix (c'est typiquement le cheminement d'Olson dans la Logique de l'action collective). On pourrait enfin envisager de considérer préférences et issues comme données, et d'explorer empiriquement le raisonnement effectif de choix, en faisant de celui-ci une variable dépendante et non plus un axiome. Mais cela est davantage le projet de la psychologie que de la théorie du choix rationnel.

La question est donc de savoir si l'axiome de rationalité fournit une approximation du comportement individuel acceptable pour expliquer ces états agrégés, dont les équilibres en théorie des jeux sont la forme élémentaire. Acceptable veut dire ici compatible avec l'observation, explicative dans un nombre de cas suffisant, et robuste par rapport à des explications concurrentes.

C'est bien là que le bât blesse. Concernant d'abord la première condition, celle de compatibilité sinon de conformité à l'observation, on peut sans doute, et dans certaines limites, amender (en interne) ou relaxer (en externe) les axiomes de rationalité, comme nous l'avons évoqué plus haut, pour intégrer une psychologie individuelle plus complexe. Ces efforts ne sont cependant pas toujours conciliables avec la modélisation formelle, parce que le comportement y est trop largement indéterminé, et ils ouvrent, quand ils le sont, à une analyse cognitive de l'intentionnalité beaucoup plus qu'à une simple validation du choix rationnel. Celui-ci invite en quelque sorte de luimême à en dépasser les limites.

L'axiome de rationalité est-il explicatif dans un nombre de cas suffisant? C'est ce que s'appliquent à réfuter D. Green et I. Shapiro dans leur bilan centré sur la politique américaine. Il y a bien des façons de concevoir cette question : le choix rationnel est-il toujours explicatif, le plus souvent explicatif, ou explicatif dans les cas les plus significatifs? Nous avons rappelé que sa validité se limite nécessairement aux conditions de risque ou assimilables. Les situations d'incertitude sont-elles plus fréquentes, donc plus importantes empiriquement, que les situations de risque, comme on le prétend souvent? On peut penser que l'incertitude, dans les contextes où les enjeux sont les plus forts, notamment les situations politiquement tendues, est appréhendée par les individus comme une situation de risque. Ma position de politiste incline plutôt en ce sens. Mais ce n'est pas certain, et difficilement évaluable. Je ne vois pas que la controverse sur le choix rationnel ait réellement permis de trancher cette question. 
Enfin, cette axiome produit-il des explications robustes par rapport à ses concurrents? Bien peu de travaux abordent de front la question. D'un côté, nombre de contributions analysent des cas empiriques en recourant à ce type d'explications. De l'autre, les critiques se focalisent pour la plupart sur l'axiomatique, sur la qualité des tests empiriques ou sur le principe de la formalisation. Mais aucune alternative théorique ne se révèle significativement plus prédictive. On est donc laissé face au choix entre une formulation théorique peu réaliste dans son axiomatique, offrant une capacité de modélisation précise et de prédiction conditionnelle, mais dont le domaine de validité est restreint, et des axiomes plus réalistes, offrant peu ou pas de capacité de modélisation et pratiquement aucun pouvoir de prédiction. Nombre d'empiristes préfèrent cette seconde position au nom de la spécificité et de la complexité du social. Mais les échecs de la grande théorie du choix rationnel ne justifient pas l'abandon de toute prétention explicative au profit d'une seule démarche idiosyncratique. Aucune démarche scientifique ne peut renoncer à la recherche de propositions dont la validité dépasse le cadre empirique de leur élaboration. Aucune théorie explicative ne peut s'abstenir de préciser les microfondations des phénomènes dont elle rend compte, c'est-à-dire les éléments et les relations dont ils sont composés. Ceci exige des modélisations, dont la dimension mathématique n'est qu'une modalité éventuelle, articulant dimensions individuelles et collectives du social dans des rapports de causalité compréhensive. Que le choix rationnel ne forme qu'un mécanisme sociologique ${ }^{11}$ parmi d'autres, dont on connaît maintenant assez bien la portée et les limites, ne doit pas détourner les sciences sociales de leur vocation : plusieurs décennies de débats invitent au contraire aujourd'hui à spécifier davantage les mécanismes et les modèles explicatifs sur lesquels elles s'appuient. L'héritage du choix rationnel est donc en-deçà de la validation globale d'une théorie, mais au-delà d'une panoplie d'instruments méthodologiques. Ses échecs rappellent la clairvoyance de Robert K. Merton sur la pertinence des théories «intermédiaires », mais exigent aussi de préciser les fondements analytiques et normatifs de ces dernières ${ }^{12}$. Ce faisant, les sciences sociales prennent un tour plus politique, plus près de «l'art du possible» dans la production de la pensée sociale et dans la Cité.

\section{BIBLIOGRAPHIE}

Ainslie, G. (1992), Picoeonomics: The Strategic Interaction of Successive Motivational States within the Person, Cambridge, Cambridge University Press.

Elster, J. (1983), Sour Grapes. Studies in the subversion of rationality, Cambridge, Cambridge University Press.

Green, D. et I. Shapiro (1994), Pathologies of Rational Choice Theory, New Haven, Yale University Press. Hedström, P. et R. Swedberg (1998), Social Mechanisms. An Analytical Approach to Social Theory, Cambridge, Cambridge University Press.

March J. G. et J. P. Olsen (1989), Rediscovering Institution, New York, The Free Press.

Needham, Joseph (1954), Science and Civilization in China, Cambridge, Cambridge University Press.

Tversky, A. et D. Kahneman (1974), «Judgement under Uncertainty», Science, nº 185, p. 1124-1130.

11. Voir notamment pour un playdoyer dans cet esprit Hedström et Swedberg (1998).

12. L'itinéraire intellectuel de James Coleman est très révélateur à cet égard. 